
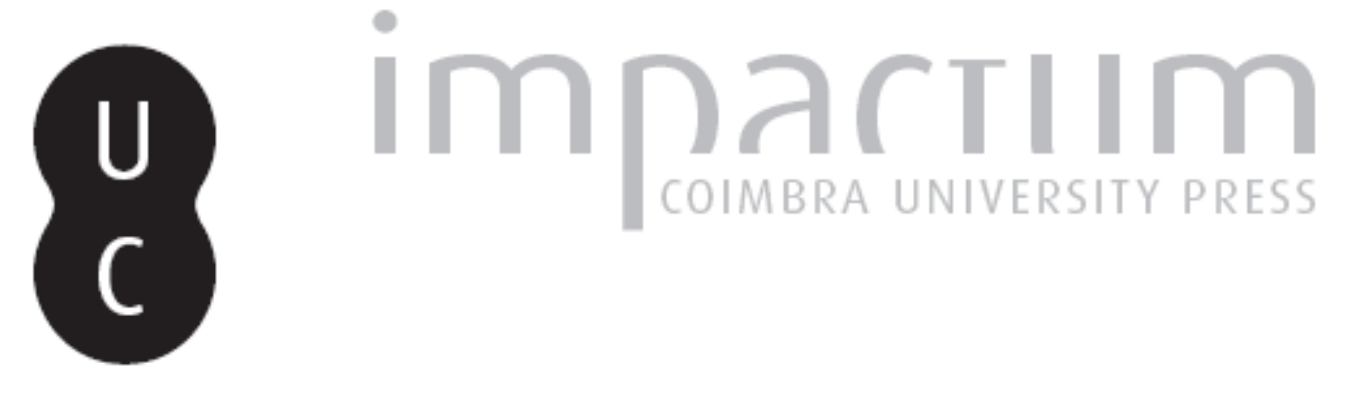

\title{
Risco de cenários de dano vulneráveis de redes de abastecimento de água
}
Autor(es): $\quad$ Pinto, Jorge; Varum, Humberto; Duarte, António; Afonso, Luís; Varajão, João; Bentes, Isabel

Publicado por: Associação Portuguesa de Riscos, Prevenção e Segurança

URL persistente:

URI:http://hdl.handle.net/10316.2/36082

DOI:

DOI:http://dx.doi.org/10.14195/1647-7723_18_26

Accessed : $\quad$ 26-Apr-2023 00:29:18

A navegação consulta e descarregamento dos títulos inseridos nas Bibliotecas Digitais UC Digitalis, UC Pombalina e UC Impactum, pressupõem a aceitação plena e sem reservas dos Termos e Condições de Uso destas Bibliotecas Digitais, disponíveis em https://digitalis.uc.pt/pt-pt/termos.

Conforme exposto nos referidos Termos e Condições de Uso, o descarregamento de títulos de acesso restrito requer uma licença válida de autorização devendo o utilizador aceder ao(s) documento(s) a partir de um endereço de IP da instituição detentora da supramencionada licença.

Ao utilizador é apenas permitido o descarregamento para uso pessoal, pelo que o emprego do(s) título(s) descarregado(s) para outro fim, designadamente comercial, carece de autorização do respetivo autor ou editor da obra.

Na medida em que todas as obras da UC Digitalis se encontram protegidas pelo Código do Direito de Autor e Direitos Conexos e demais legislação aplicável, toda a cópia, parcial ou total, deste documento, nos casos em que é legalmente admitida, deverá conter ou fazer-se acompanhar por este aviso.

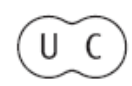




\section{territorium}

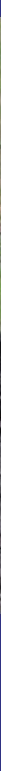

\section{Afirmar as Ciências Cindínicas}

Revista da Associação Portuguesa de Riscos, Prevenção e Segurança 
Jorge Pinto Departamento de Engenharias, ECT, Universidade de Trás-os-Montes e Alto Douro tiago@utad.pt

Humberto Varum Departamento de Engenharia Civil, Universidade de Aveiro hvarum@ua.pt

António Duarte Departamento de Engenharia Civil, Universidade do Minho aduarte@civil.uminho.pt

Luís Afonso lu.afonso@gmail.com

João Varajão jvarajao@utad.pt

Isabel Bentes Departamento de Engenharias, ECT, Universidade de Trás-os-Montes e Alto Douro ibentes@utad.pt

\section{RESUMO}

Neste trabalho propõe-se uma metodologia integrada para a quantificação do risco associado a cenários de dano de redes de abastecimento de água, baseada na teoria da vulnerabilidade, com quantificação das consequências associadas às perdas da rede, para cada cenário identificado. A identificação das vulnerabilidades e dos riscos inerentes a este tipo de sistemas poderão contribuir para os tornar mais robustos, melhorando a qualidade do seu funcionamento, e diminuindo o potencial impacto de um cenário de dano nos utilizadores.

Palavras chave: risco em sistemas de abastecimento de água, gestão patrimonial de infra-estruturas, teoria da vulnerabilidade, cenários de dano.

\section{RESUMEN}

Riesgo de escenarios de daño vulnerables de redes de distribución de agua - En este trabajo se propone una metodología integrada para la cuantificación del riesgo asociado a escenarios de daño de redes de distribución de agua, basada en la teoría de vulnerabilidad, con cuantificación de las consecuencias asociadas a la perdida de la red, para cada escenario identificado. La identificación de vulnerabilidades e de riesgos asociados a este tipo de sistemas podrá contribuir para tornarlos más robustos, disminuyendo el potencial impacto de cada escenario de daño.

Palabras clave: riesgo en sistemas de distribución de agua, gestión de infraestructuras, teoría de la vulnerabilidad, escenarios de daño.

\section{RESUMÉ}

Risque de scénarios vulnérables de systèmes de fourniture de l'eau - Dans ce travail est présenté une méthodologie intégrée visant mesurer le risque associé à scénarios de dommage de réseaux d'approvisionnement de l'eau. Cette méthodologie est basée sur la théorie de la vulnérabilité et permit de quantifier les conséquences associées aux pertes du réseau, pour chaque scénario identifié. L'identification des vulnérabilités de systèmes pourra contribuer pour qu'ils deviennent plus robuste et diminuer l'impact chez les utilisateurs en cas de dommage.

Mots-clé: risque en systèmes de fourniture de l'eau de, gestion de infrastructures, théorie de la vulnérabilité, scénarios de dommage.

\section{ABSTRACT}

Risk of damage scenarios in vulnerable water supply networks - This work proposes an integrated methodology for the quantification of the risk associated to damage scenarios in water supply networks, based on the theory of vulnerability, with the quantification of the consequences associated to the loss of the network, for each identified scenario. The identification of the vulnerabilities can contribute to make them more robust as well as to reduce the potential impact of a damage scenario for the system's users.

Key words: risks in water supply systems, infrastructures' management, theory of vulnerability, damage scenarios.

* O texto deste artigo corresponde à comunicação apresentada ao II Congresso Internacional de Riscos e VI Encontro Nacional, tendo sido submetido para revisão em 07-06-2010, tendo sido aceite para publicação em 12-08-2010.

Este artigo é parte integrante da Revista Territorium, n. ${ }^{\circ} 18,2011$, ${ }^{\circledR}$ RIscos, ISBN: 0872- 8941. 


\section{Introdução}

Vulnerabilidade, susceptibilidade, dano e risco são alguns termos frequentemente usados no nosso quotidiano nos mais diversos quadrantes da sociedade. Estão associados a situações que colocam em risco a salvaguarda da segurança e do bem-estar dos cidadãos e dos seus bens.

Risco de incêndio, risco natural, risco geomorfológico, risco de movimento de massa, risco de ravinamento, risco de erosão, risco em bacias hidrográficas, risco ambiental, risco de ondas de calor, risco de transporte de matérias químicas perigosas, risco em áreas urbanas degradadas, risco geotécnico, risco sísmico-vulcânico, risco à catástrofe, risco para a saúde, risco de exposição a agentes químicos, risco de navegação, risco de contaminação, entre outros, são contextos de estudo sobre os quais a comunidade científica, nacional e internacional, têm vindo a investir de forma a apresentar soluções capazes de proteger as populações e o meio ambiente (RIscos, 2010).

As causas naturais (i.e. sismos, erupções vulcânicas, incêndios, cheias, secas, temperaturas extremas, tornados, deslocamento de massas, entre outras), os acidentes (i.e. derrame de petróleo, contaminação nuclear, poluição da água de um rio, contaminação da água de uma rede pública de abastecimento, queda de um avião, incêndio num edifício, entre outros), os actos de negligência, os actos criminosos e os actos terroristas constituem-se como um conjunto vasto de cenários de risco que podem implicar globalmente um elevado nível de insegurança.

O estudo de mecanismos de redução desta insegurança é fundamental e urgente. É unânime que este estudo é muito complexo porque necessita de integrar aspectos tão distintos como ambientais, económicos, políticos, culturais, entre outros tornando por isso o conceito de risco multidimensional ( $F$. Rebelo, 2009). Este facto requer por isso que a comunidade científica se una de forma a criar equipas de investigação multidisciplinares capazes de lidar com esta complexidade.

Diversos trabalhos de investigação têm vindo a ser desenvolvidos de forma a reduzir a vulnerabilidade inerente a diversos sistemas e em diversos contextos ( $\mathrm{J}$. T. PINTO et al., 2002; X. Wu et al., 1993; J. T. PINTO et al., 2010-c; J. T. PINTO et al., 2010-a; J. T. PInTo et al., 2010b; D. McEntiRe et al., 2010). A correcta compreensão dos factores de vulnerabilidade é fundamental por forma a definir rigorosamente as acções mais adequadas para a redução do risco (J. T. PINTO et al., 2002).

Neste contexto, a teoria da vulnerabilidade de redes de abastecimento de água (TVRAA) tem como principal objectivo identificar os elementos mais vulneráveis de um sistema desta natureza através da identificação de diferentes tipos de cenários de dano, sendo o cenário de máxima vulnerabilidade aquele em que um pequeno esforço origina um dano desproporcional (J. T. PINTo et al., 2010-a; J. T. PINTo et al., 2010-b). Nesta teoria, as acções que podem provocar o dano na rede de abastecimento de água (RAA) podem ser de vários tipos, tais como: excesso de pressão hidráulica, envelhecimento das condutas, agressividade do meio envolvente, erro de projecto, erro de construção, sabotagem, alteração da qualidade da água, entre outras. 0 dano é considerado como sendo uma perda da RAA ou de parte da RAA, correspondente a um ou mais troços. Esta teoria utiliza a construção de um modelo hierárquico e, como tal, não necessita de estudar todas as combinações possíveis de danos na RAA.

A teoria terá uma maior aplicabilidade em sistemas emalhados ou mistos (i.e. partes emalhadas e partes ramificadas). A aplicação da teoria a sistemas ramificados poderá conduzir a resultados previsíveis reduzindo por isso a sua relevância.

Este artigo está estruturado da seguinte forma. Inicialmente, a TVRAA é apresentada e descrita de uma forma sucinta, onde os principais conceitos teóricos serão evidenciados. Esta tarefa será complementada com o recurso a um exemplo simples de aplicação a uma RAA. Seguidamente, será proposta uma metodologia expedita de mapeamento da vulnerabilidade de RAA e uma metodologia de quantificação do risco de danos vulneráveis neste tipo de sistemas. Finalmente, serão expostas as principais conclusões e elaboradas algumas considerações finais.

\section{Breve descrição da TVRAA}

\section{Conceitos teóricos mais relevantes}

Para uma melhor familiarização com a TVRAAé importante conhecer alguns dos conceitos que constituem a sua base teórica, que se apresentam seguidamente:

- Sub-RAA primitiva é constituída por um troço e os seus respectivos nós.

- Sub-RAA é entendida como sendo um agrupamento de pelo menos dois troços adjacentes e dos respectivos nós.

- Sub-RAA de referência é o(s) reservatório(s).

- Sub-RAA de origem entende-se como sendo toda a RAA, incluindo o reservatório.

- Anel de RAA é uma forma abstracta de representar uma RAA. Este poderá ser aberto ou fechado caso a RAA seja ramificada ou emalhada respectivamente, nessa zona. 
- Dano de RAA ou evento de deterioração corresponde a uma deterioração da RAA que pode impossibilitar o adequado abastecimento de água com qualidade entre pontos dessa RAA. Qualidade de forma é um indicador da boa forma de uma RAA ou de uma sub-RAA.

- Conexão nodal é o indicador da interligação de uma sub-RAA à restante RAA.

- Capacidade resistente ao dano é uma grandeza indicadora da capacidade da RAA resistir a esforços. Nesta fase, a capacidade resistente ao dano tem sido considerada igual à pressão de serviço.

- Cenário de dano é uma sequência ordenada de danos da RAA (ou de eventos de deterioração) através dos quais o adequado nível de desempenho da RAA não é garantido.

- Capacidade relativa de resistência ao dano é entendida como sendo a relação entre o esforço requerido para a ocorrência de um cenário de dano e o esforço necessário para a ocorrência do cenário de dano total da RAA.

- Perda de RAA é uma grandeza que traduz a parte de uma RAA que fica inoperável, resultante da ocorrência de um cenário de dano.

\section{- Aplicação da TVRAA}

O desenvolvimento da TVRAA até à identificação dos cenários de dano é feito em três etapas, à semelhança da aplicação da teoria da vulnerabilidade estrutural (J. T. PInTo et al., 2010-b). A primeira etapa consiste no processo de aglutinação, a segunda etapa é a representação da RAA através de um modelo hierárquico e, a terceira e última etapa é relativa ao processo de desaglutinação desse modelo hierárquico.

0 processo de aglutinação é um processo selectivo e iterativo através do qual uma RAA vai sendo progressivamente aglutinada. Esta aglutinação vai sendo processada tendo em conta diferentes aspectos dos quais se destaca a qualidade de forma da RAA. Para o efeito, são usados cincos critérios de aglutinação que são os seguintes por ordem de importância: menor perda de carga total $\left(\Delta \mathrm{H}_{\text {min }}\right)$; máxima capacidade resistente ao dano $\left(\mathrm{E}_{\max }\right)$; máxima conexão nodal $\left(\mathrm{n}_{\max }\right)$; máxima distância ao reservatório ( $\left.\mathrm{DiS}_{\max }\right)$; e, escolha Livre $\left(\mathrm{F}_{c}\right)$. Com a informação resultante do processo de aglutinação é possível traçar o modelo hierárquico da RAA, que corresponde a uma representação abstracta da RAA em que esta se encontra rearranjada em termos de forma. Finalmente, usando este modelo hierárquico e sete critérios de selecção o processo de desaglutinação permite identificar os cenários de dano vulneráveis. Este processo de desaglutinação também é selectivo, iterativo e interactivo porque requer que novos processos de aglutinação sejam processados à medida que os eventos de dano vão sendo identificados, até que a RAA ou a sub-RAA em análise fique totalmente inoperável. Os critérios de selecção do processo de desaglutinação são por ordem de importância: a sub-RAA não é uma sub-RAA de referência $\left(\mathrm{N}_{\mathrm{R}}\right)$; a sub-RAA está ligada directamente à sub-RAA de referência $\left(C_{D}\right)$; seleccionar uma sub-RAA primitiva (troço) em detrimento de uma sub-RAA $\left(L_{c}\right)$; sub-RAA apresenta o maior valor de perda de carga total $\left(\mathrm{S}_{\Delta \mathrm{H}}\right)$; a sub-RAA tem o menor valor de capacidade resistente ao dano $\left(\mathrm{S}_{\mathrm{E}}\right)$; a sub-RAA foi aglutinada posteriormente $\left(C_{L}\right)$; escolha livre $\left(F_{c}\right)$. Em trabalhos anteriores (J. T. PINTO et al., 2010-a; J. T. PINTO et al., 2010-b), os processos de aglutinação e de desaglutinação foram já detalhadamente descritos.

\section{Cenários de dano vulneráveis}

A TVRAA identifica cinco tipos de cenários de dano, que são: o cenário de colapso total, o cenário de máxima vulnerabilidade, o cenário de mínima vulnerabilidade, o cenário de menor esforço para haver dano e o cenário de dano de interesse. 0 cenário de colapso total é o cenário de dano que corresponde à perda da totalidade de uma RAA, ou seja em que toda a RAA fica incapacitada de abastecer água a qualquer ponto. 0 cenário de colapso total será aquele que, dentro de todos os cenários de dano que apresentem um valor de perda de RAA unitário, tenha o maior valor do índice de vulnerabilidade. o cenário de máxima vulnerabilidade é aquele que apresenta a maior desproporcionalidade entre a perda de RAA e o esforço, e por isso apresenta o maior valor do índice de vulnerabilidade. Este cenário está associado à parte mais vulnerável de uma RAA. Por sua vez, o cenário de mínima vulnerabilidade é aquele que conduz à menor perda da RAA, e geralmente corresponde ao dano da última sub-RAA primitiva que foi aglutinada. 0 cenário de menor esforço para haver dano é aquele que corresponde ao elemento da RAA que requer o menor esforço para sofrer dano. Finalmente, o cenário de dano de interesse é um cenário de dano específico cujo utilizador da TVRAA pretenda analisar por algum motivo particular (implicações económicas, abastecimento a estrutura de maior importância, implicações em outras infra-estruturas, etc.).

\section{Exemplo de aplicação da TVRAA}

De forma a exemplificar a aplicação da TVRAA, e a complementar a descrição sucinta dos conceitos teóricos desenvolvida anteriormente, recorre-se à RAA ilustrada na fig. 1. Esta RAA é formada por quatro troços (troços numerados de 1 a 4) e por cinco nós (nós numerados de 1 a 5). 0 reservatório é designado por 5 . Esta RAA é propositadamente simples para simplificar a aplicação aqui desenvolvida e facilitar a compreensão dos 
conceitos inerentes a esta teoria. O QUADRo I apresenta algumas características geométricas e hidráulicas da RAA analisada.

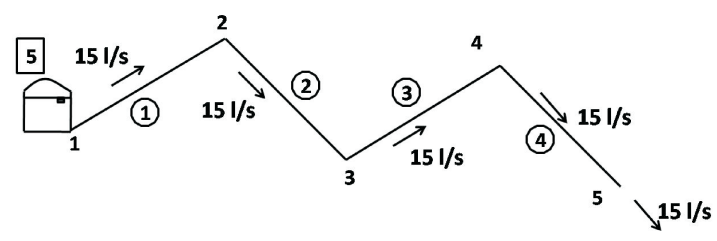

Fig. 1 - RAA usada como exemplo (planta) (Fonte: Autores).

QUADRO I - Características geométricas e hidráulicas da RAA.

\begin{tabular}{|c|c|c|c|c|c|c|}
\hline Troço & Nós & $\begin{array}{c}\text { Comprimento } \\
(\mathrm{m})\end{array}$ & $\begin{array}{c}\text { Caudal } \\
(\mathrm{l} / \mathrm{s})\end{array}$ & $\begin{array}{c}\text { Diâmetro } \\
\text { Comercial } \\
(\mathrm{mm})\end{array}$ & $\begin{array}{c}\text { Perda } \\
\text { de } \\
\text { carga } \\
\text { total } \\
(\text { m.c.a. })\end{array}$ & $\begin{array}{c}\text { Capacidade } \\
\text { resistente } \\
\text { ao dano } \\
\left(\mathrm{kgf} / \mathrm{cm}^{2}\right)\end{array}$ \\
\hline 1 & $1 ; 2$ & 200 & 15 & 160 & 0,84 & 6 \\
\hline 2 & $2 ; 3$ & 200 & 15 & 160 & 0,84 & 6 \\
\hline 3 & $3 ; 4$ & 200 & 15 & 160 & 0,84 & 6 \\
\hline 4 & $4 ; 5$ & 200 & 15 & 160 & 0,84 & 6 \\
\hline
\end{tabular}

Toda a RAA estudada é formada por tubos de policloreto de vinilo (PVC) com um valor de rugosidade absoluta equivalente (adiante designada por rugosidade) de 0,01 mm. O valor da viscosidade cinemática da água foi considerado igual a 1,01 x 10-6 m²/s. A pressão de serviço adoptada para o dimensionamento hidráulico da referida RAA foi de $6 \mathrm{kgf} / \mathrm{cm}^{2}$. Por sua vez, o QUADRo II apresenta os valores de altimetria dos nós a montante (M) e a jusante $(\mathrm{J})$ de cada troço da RAA.

\section{QUADRO II - Quotas.}

\begin{tabular}{|c|c|c|c|c|c|c|}
\hline \multirow{2}{*}{ Troço } & \multicolumn{2}{|c|}{$\begin{array}{c}\text { Cota topográfica } \\
(\mathrm{m})\end{array}$} & \multicolumn{2}{c|}{$\begin{array}{c}\text { Cota } \\
\text { piezométrica } \\
\text { (m.c.a.) }\end{array}$} & \multicolumn{2}{c|}{$\begin{array}{c}\text { Altura } \\
\text { piezométrica } \\
\text { (m.c.a.) }\end{array}$} \\
\cline { 2 - 7 } & $\mathrm{M}$ & $\mathrm{J}$ & $\mathrm{M}$ & $\mathrm{J}$ & $\mathrm{M}$ & $\mathrm{J}$ \\
\hline 1 & 163,68 & 130,00 & 293,68 & 165,84 & 3,00 & 35,84 \\
\hline 2 & 130,00 & 140,00 & 270,00 & 165,00 & 35,84 & 25,00 \\
\hline 3 & 140,00 & 130,00 & 270,00 & 164,16 & 25,00 & 34,16 \\
\hline 4 & 130,00 & 135,00 & 265,00 & 163,32 & 34,16 & 28,32 \\
\hline
\end{tabular}

Exemplificação da aplicação do processo de aglutinação e apresentação do modelo hierárquico resultante

Na RAA existem três candidatos de sub-RAA primitivas passíveis de serem aglutinados no primeiro passo. Para todos os candidatos calculam-se os parâmetros de vulnerabilidade de perda de carga total, de capacidade resistente ao dano, de conexão nodal e de distância ao reservatório.

Aplicando-se os critérios de selecção do processo de aglutinação verifica-se que o primeiro candidato a formar uma nova sub-RAA corresponde à aglutinação das sub-RAA primitivas 2 e 3 , pois é o candidato que apresenta o maior valor de conexão nodal dando origem à nova sub-RAA 6 .

O segundo passo consiste em aglutinar as sub-RAA 4 e 6, e o critério de selecção aplicado foi o de máxima distância ao reservatório.

No terceiro passo, resta apenas um candidato independente da sub-RAA de referência para ser aglutinado (o candidato formado pelas sub-RAA 1 e 7 ) e que é agora seleccionado.

0 processo termina com a aglutinação da sub-RAA de referência 5 com a restante RAA já aglutinada dando origem à sub-RAA 8. Todo este processo de aglutinação está descrito esquematicamente na fig. 2. 0 modelo hierárquico resultante deste processo está apresentado na fig. 3-a.

Exemplificação da aplicação do processo de desaglutinação e cenários de dano vulneráveis identificados

A informação resultante da aplicação do processo de aglutinação é fundamental para o desenrolar do processo de desaglutinação. Todas as sub-RAA geradas no processo de aglutinação vão ser analisadas individualmente através de um desmembramento assente nos critérios de selecção e na busca de eventos de dano. Esta análise individual termina quando os eventos de dano identificados tornam a sub-RAA ou a RAA inoperáveis. A fig. 3 mostra esquematicamente o desenrolar da aplicação do processo de desaglutinação à RAA usada como exemplo. A numeração das sub-RAA existentes nos modelos hierárquicos da fig. 3 não corresponde integralmente à numeração da fig. 2, devido aos sucessivos processos de aglutinação intercalares necessários durante o processo de desaglutinação.

\section{Cenários de dano vulneráveis resultantes}

Através do processo de desaglutinação foram identificados dois cenários de dano vulneráveis, o primeiro corresponde ao dano no troço 1 (T1) e o segundo ao dano no troço 2 (T2).

O cenário de colapso total corresponde ao primeiro cenário de dano vulnerável identificado, dano no troço 1 (T1) e porque é o que corresponde à total perda da RAA. Se o troço 1 sofrer dano então a RAA fica incapaz de abastecer água com qualidade a qualquer ponto. Este cenário é o esperado, o que em parte valida a TVRAA.

O cenário de máxima vulnerabilidade também é o primeiro cenário de dano vulnerável identificado, T1, uma vez que é aquele que apresenta o maior valor do 

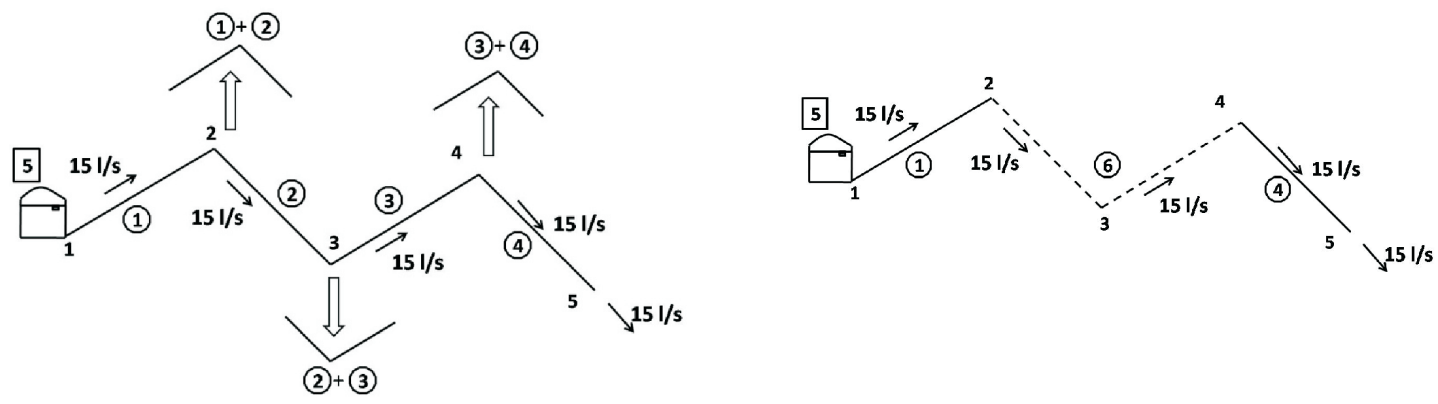

a) $1^{\circ}$ Passo: Formação da sub-RAA 6

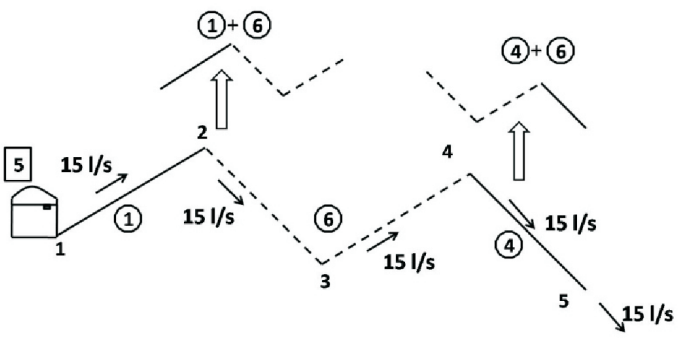

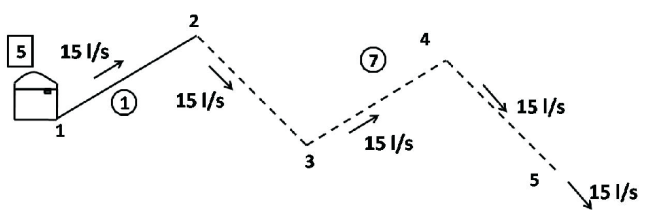

b) $2^{\circ}$ Passo: Formação da sub-RAA 7
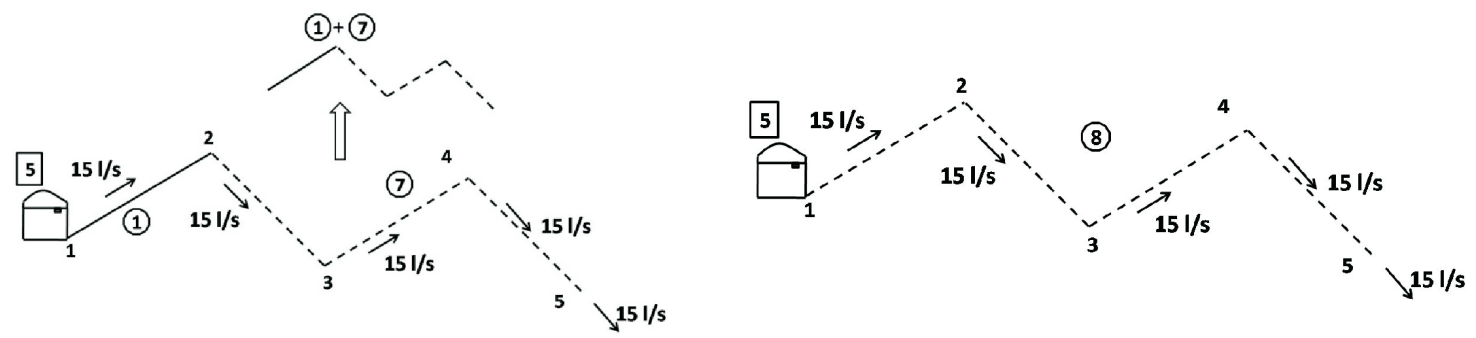

c) $3^{\circ}$ Passo: Formação da sub-RAA 8

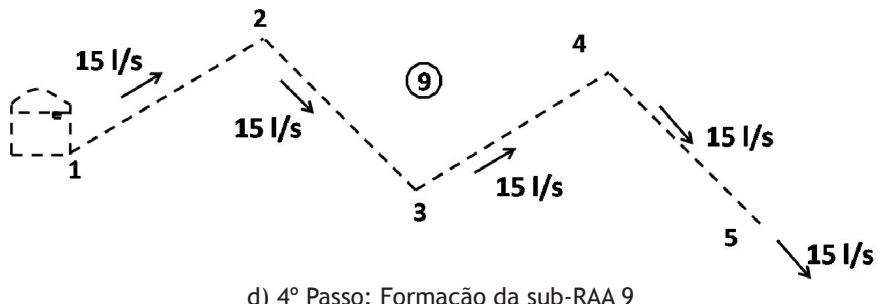

Fig. 2 - Descrição esquemática do processo de aglutinação da RAA usada como exemplo (Fonte: Autores).

índice de vulnerabilidade. Este resultado também valida a TVRAA porque é evidente que é requerido o mesmo esforço para provocar dano nos troços 1 e 2, porque ambos têm igual pressão de serviço $\left(6 \mathrm{kgf} / \mathrm{cm}^{2}\right)$. Também é evidente que a consequência desses danos em termos de perda da RAA é diferente.

O cenário de mínima vulnerabilidade é aquele que corresponde à ocorrência de dano no troço 1 (T1), porque este troço foi o último a ser aglutinado, fig. 3-a. Este cenário aparentemente não é evidente. Contudo, tendo em conta a particularidade das características hidráulicas da RAA percebe-se que a zona da rede relativa ao troço 1 apresenta, de facto, a menor qualidade de forma porque os factores de interligação entre sub-RAA e distância ao reservatório são relevantes neste caso. 0 facto do troço 1 estar ligado directamente ao reservatório implica que o impacto no bom funcionamento da RAA devido à ocorrência de um dano nesse troço seria claramente mais expressivo do que o respectivo impacto resultante 


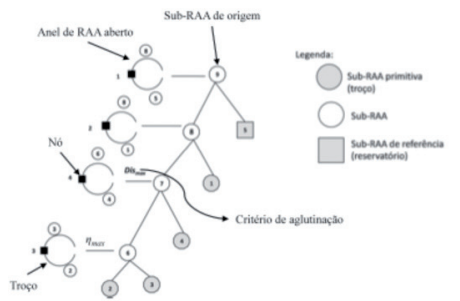

a) Modelo hierárquico da sub-RAA 9

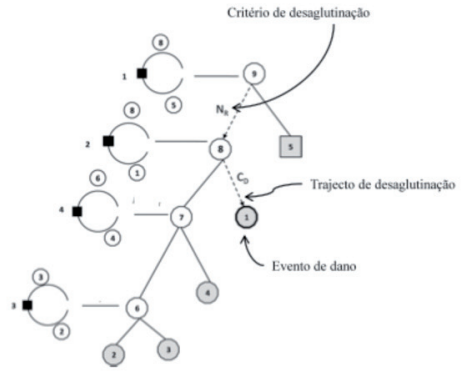

b) Desaglutinação da sub-RAA 9

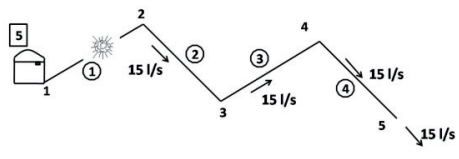

c) Cenário de dano T1

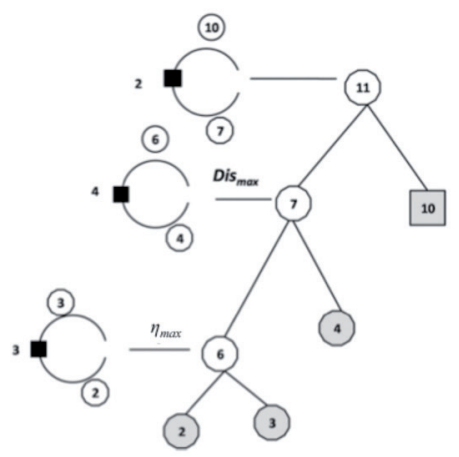

d) Modelo hierárquico da sub-RAA 7

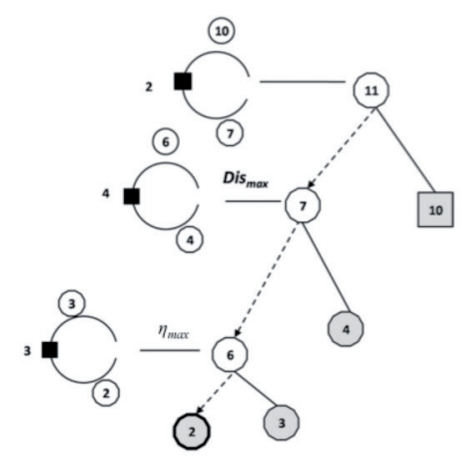

e) Desaglutinação da sub-RAA 7

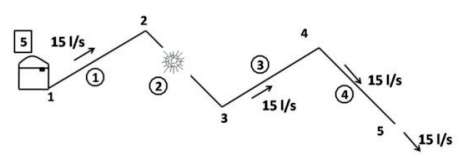

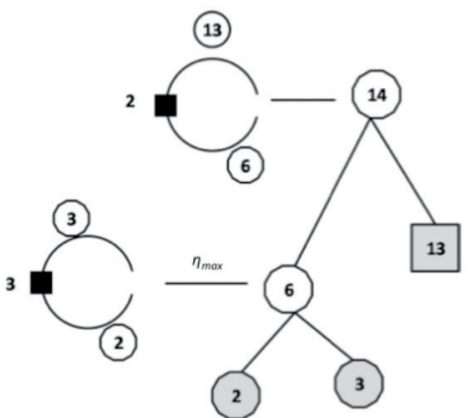

g) Modelo hierárquico da sub-RAA 6

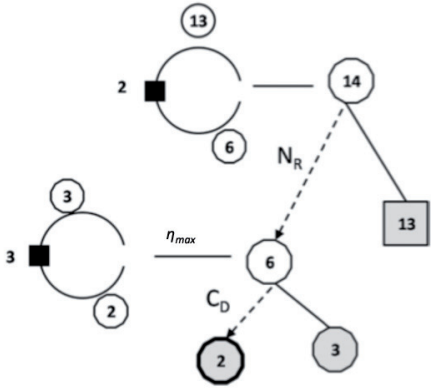

h) Desaglutinação da sub-RAA 6
Igual a f)

i) Cenário de dano T2

Fig. 3 - Processo de desaglutinação da RAA (Fonte: Autores).

da ocorrência de dano em outro troço (T4, por exemplo) que se encontra mais afastado do reservatório.

O cenário correspondente ao menor esforço que provoca dano poderá estar associado à ocorrência de dano em qualquer troço da RAA, porque todos os troços apresentam o mesmo valor de capacidade resistente ao dano, QuadRo I. Deste modo, este cenário pode ser dano em T1, ou T2, ou T3, ou T4.

\section{Mapeamento da vulnerabilidade de uma RAA}

O mapeamento da vulnerabilidade de uma RAA poderá ser um elemento de trabalho muito útil para a gestão de sistemas deste tipo. A metodologia de mapeamento da vulnerabilidade de RAA proposta neste trabalho de investigação recorre a uma escala relativa de vulnerabilidade que contempla três graus de vulnerabilidade. Esses graus são "Muito vulnerável", "Vulnerável" e "Pouco vulnerável".

A escala de vulnerabilidade proposta é relativa, porque os valores do índice de vulnerabilidade resultantes da TVRAA são específicos de uma determinada RAA, não estando prevista a sua utilização na comparação da vulnerabilidade entre RAA diferentes.

A escala relativa de vulnerabilidade de uma RAA é obtida usando como base o maior e o menor valor de índice de vulnerabilidade $\left(\varphi_{\text {maior }}\right.$ e $\varphi_{\text {menor }}$, respectivamente) dos cenários de dano vulneráveis identificados através da aplicação da TVRAA. O intervalo de vulnerabilidade (A, fig. 4) é calculado através da diferença desses valores. Esse intervalo de vulnerabilidade é subdividido em três intervalos iguais, correspondendo cada um a um dos 
graus de vulnerabilidade identificados anteriormente, tal como mostra a fig. 4 .

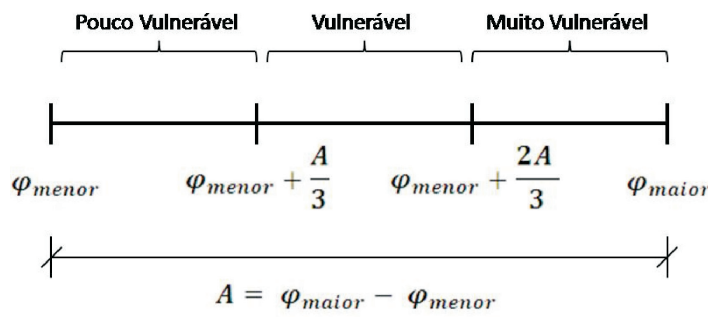

Fig. 4 - Quantificação dos intervalos da escala relativa de vulnerabilidade (Fonte: Autores).

Face ao exposto e aos resultados obtidos através da TVRAA é possível mapear a vulnerabilidade da RAA usada como exemplo (apresentada no capítulo anterior), tal como se ilustra na fig. 5 .

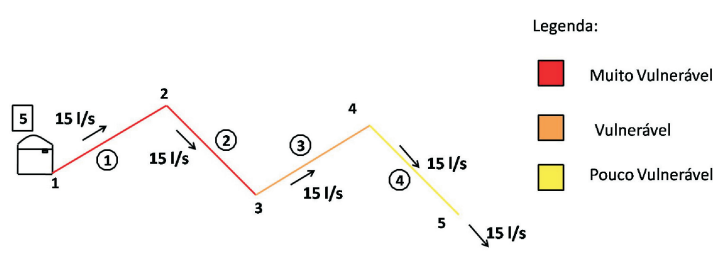

Fig. 5 - Mapeamento da vulnerabilidade da RAA (Fonte: Autores).

\section{Quantificação do risco de danos vulneráveis}

De acordo com D. I. BLockley (1999), risco é definido como sendo o efeito combinado da possibilidade de ocorrência e da consequência resultante de um desastre, num determinado contexto. Inspirado nesta definição foi proposta por J. T. Pinto et al. (2002) uma metodologia para a quantificação do risco de danos vulneráveis em estruturas. No contexto do bom funcionamento de uma RAA, a metodologia de quantificação do risco associado aos cenários de dano vulneráveis passíveis de ocorrer em RAA (WR), proposta neste trabalho de investigação, baseia-se na proposta anteriormente desenvolvida para estruturas (J. T. PINTO et al., 2002), e que correspondente à aplicação da seguinte expressão:

$$
\mathrm{WR}=\mathrm{pfw} \times \mathrm{WCon}
$$

em que pfw é a probabilidade de ocorrência de um cenário de dano vulnerável e WCon é a consequência em termos de dano no funcionamento da RAA resultante da ocorrência desse cenário. Os cenários de dano vulneráveis são identificados através da TVRAA. WCon é função da perda de RAA que é quantificada também através da TVRAA.

\section{Conclusões}

A TVRAA foi sucintamente apresentada e os seus principais conceitos teóricos foram também elencados e descritos. A aplicação desta teoria é mais relevante em sistemas emalhados ou mistos (i.e. resultantes da combinados de sistemas ramificados e emalhados).

Um exemplo simples de uma RAA foi usado de forma a facilitar a exposição dos conceitos e estratégia envolvidos, permitindo ainda ilustrar a aplicação da TVRAA e mostrar seu o potencial. Este exemplo simples permitiu validar a teoria uma vez que conduz a resultados expectáveis, isto é que os cenários de colapso total, de máxima vulnerabilidade, de mínima vulnerabilidade e de menor esforço para haver dano são todos iguais, e correspondem ao dano no troço 1 da RAA. Por sua vez, os vários critérios de aglutinação foram aplicados neste exemplo, contrastando com outros exemplos anteriormente analisados (J. T. PINTO et al., 2010-c; J. T. PInTo et al., 2010-a; J. T. PInTo et al., 2010-b), onde apenas o primeiro critério (menor perda de carga total) foi aplicado.

Foi proposta uma forma expedita de mapeamento da vulnerabilidade de uma RAA. Esta ferramenta poderá ser extremamente útil na gestão de um sistema deste tipo.

Uma metodologia de quantificação do risco de danos vulneráveis de RAA também foi proposta neste trabalho de investigação. Esta metodologia foi inspirada numa outra desenvolvida anteriormente no contexto das estruturas. Nesta metodologia, a TVRAA desempenha um papel fundamental porque não só identifica os cenários de dano vulneráveis como também permite quantificar a perda de RAA associada. Esta grandeza facilitará o cálculo da consequência de determinado dano no bom funcionamento da RAA. Contudo, a tarefa de quantificação da probabilidade de ocorrência de um cenário de dano vulnerável é complexa, atendendo a todas as incertezas associadas e, nesta fase, ainda requer trabalho de investigação adicional.

Os conceitos e as metodologias aqui apresentados poderão ser extrapolados para outros tipos de sistemas. A identificação da vulnerabilidade destes sistemas e a quantificação do risco associado poderão contribuir para os tornar mais robustos através de um processo de mitigação do risco. Este procedimento permitirá contribuir para a segurança das populações, a salvaguarda de bens e para a redução de prejuízos económicos.

\section{Referências bibliográficas:}

RISCOS (2010) - Afirmar as Ciências Cindínicas: Reequacionar o Conhecimento dos Riscos e das Catástrofes. Livro de Resumos do II Congresso Internacional e VI Encontro Nacional de Riscos, Edição RISCOS - Associação Portuguesa de Riscos, Prevenção e Segurança, ISBN: 978-98996253-1-0, Coimbra; 
BLoCKLEY, D. I. (1999) - "Risk based structural safety methods in context", Vol. 21, n. ${ }^{\circ} 4$, “Journal of Structural Safety”, p. 335-348;

McEntire, D.; Croker, C. e Peters, E. (2010) - “Addressing vulnerability through an integrated approach", Vol. 1, n. ${ }^{\circ}$ 1, "International Journal of Distaster Resillience in the Built Environment”, p. 50-64;

Pinto, J. T.; Afonso, L.; Varajão, J. e Bentes, I. (2010a) - "Increasing the quality of water pipe network systems by tracing the vulnerability". Proceedings of the WSEAS International Conferences, Editors: Ali Kallel, Abdelhamid Hassairi, Cornelia Bulucea, Nikos Mastorakis, ISBN: 978-960-474-191-5. ISSN: 1790-5095, Kantaoui, Sousse, Tunisia;

Pinto, J. T.; Blockley, D. I. e Woodman, N. (2002) - "The risk of a vulnerable failure", Vol. 24, “Journal of Structural Safety", pp. 107-122;
Pinto, J. T.; Varum, H.; Bentes, I. e Agarwal, J. (2010b) - "A Theory of Vulnerability of Water Pipe Network (TVWPN)", "Water Resources Management" (in press, available online at http://www.springerlink.com/content/ x024016077428716/);

Pinto, J.; Varum, H.; Varajão, J. e Bentes, I. (2010-c) - “Oil and gas pipeline distribution networks: A new approach to access their vulnerability and risk". Proceedings of the Conference Pipeline Construction, Operation \& Maintenance, Doha, Qatar;

Rebelo, F. (2009) - "Nota de Abertura”, Territorium, n. ${ }^{\circ}$ 16, "Revista da Associação Portuguesa de Riscos, Prevenção e Segurança”, Edição Riscos, ISBN: 0872-8941, p. 3-4;

Wu, X.; Blockley, D. I. e Woodman N. J. (1993) "Vulnerability analysis of structural systems, Part I: Rings and clusters, Part II: Failure scenario", Vol. 10, “Jornal of Civil Engineering Systems”, p. 301-333. 